DOI: 10.1515/awutp -2015-0009

\title{
A NOTE ON THE FIRST CLASS APPROACHES OF MASSIVE P-FORMS
}

\author{
S. C. Sararu, M. T. Udristioiu
}

Department of Physics, University of Craiova, 13 Al. I. Cuza Str., Craiova 200585, Romania

\section{Article Info}

Received: 28 December 2011

Accepted: 19 January 2012

Keywords: quantization methods, path integral.

\begin{abstract}
The problem of quantization of the massive $p$-forms is considered in the framework of the Batalin-Fradkin (BF) approach based on path integral. The quantization procedure relies on the path integral of a first-class system equivalent with the original theory. This first-class system is constructed using the BF method.
\end{abstract}

\section{Introduction}

In this paper we approach the problem of the Hamiltonian quantization of the massive $p$-forms. In view of this, we construct a first-class system equivalent with the original second-class theory using the BF method [1]-[4] and then we quantify the resulting first-class system. The associated first-class system has to satisfy the following requirements: its number of physical degrees of freedom coincides with that of the original second-class theory and the algebras of classical observables are isomorphic.

\section{BF Method}

We start with a bosonic dynamic system with the phase-space locally parameterized by $n$ canonical pairs $z^{a}=\left(q^{i}, p_{i}\right)$, endowed with the canonical Hamiltonian $H_{c}$, and subject to the second-class constraints

$$
\chi_{\alpha_{0}}(z) \approx 0, \quad \alpha_{0}=\overline{1,2 M_{0}} .
$$

In order to construct a first-class system equivalent to the starting second-class one (subject to the second-class constraints (1)) in the framework of the BF approach we enlarge the original phase-space with the variables $\left(\zeta^{\alpha}\right)_{\alpha=\overline{1,2 M}},\left(M \geq M_{0}\right)$ and extend the Poisson bracket to the 
newly added variables. The next step is to construct a set of independent, smooth, real functions defined on the extended phase-space, $\left(G_{A}(z, \zeta)\right)_{A=\overline{1, M_{0}+M}}$, such that

$$
G_{\alpha_{0}}(z, 0) \equiv \chi_{\alpha_{0}}(z), \quad G_{\bar{A}}(z, 0) \equiv 0, \quad\left[G_{A}, G_{B}\right]=0,
$$

where $\bar{A}=\overline{2 M_{0}+1, M_{0}+M}$. In the last step we generate a smooth, real function $H_{\mathrm{BF}}(z, \zeta)$, defined on the extended phase-space, with the properties

$$
H_{\mathrm{BF}}(z, 0) \equiv H_{c}(z), \quad\left[H_{\mathrm{BF}}, G_{A}\right]=V_{A}^{B} G_{B} .
$$

The previous steps unravel a dynamic system subject to the first-class constraints $G_{A}(z, \zeta) \approx 0$ and whose evolution is governed by the first-class Hamiltonian $H_{\mathrm{BF}}(z, \zeta)$. The first-class system constructed by the BF method is classically equivalent to the original second-class theory since both display the same number of physical degrees of freedom

$$
\mathrm{N}_{\mathrm{BF}}=\frac{1}{2}\left[2 n+2 M-2\left(M_{0}+M\right)\right]=\frac{1}{2}\left(2 n-2 M_{0}\right)=\mathrm{N}_{O}
$$

and the corresponding algebras of classical observables are isomorphic. Consequently, the two systems become also equivalent at the level of the path integral quantization and we can to replace the Hamiltonian path integral of the original second-class theory with that of the BF first-class system.

\section{Massive p-forms}

Massive $p$-forms in $D$ space-time dimensions $(D>(p+1))$ are described by the Lagrangian action [5]

$$
S_{0}^{L}\left[A_{\mu_{1} \ldots \mu_{p}}\right]=\int d^{D} x\left(-\frac{1}{2 \cdot(p+1) !} F_{\mu_{1} \ldots \mu_{p+1}} F^{\mu_{1} \ldots \mu_{p+1}}-\frac{m^{2}}{2 \cdot p !} A_{\mu_{1} \ldots \mu_{p}} A^{\mu_{1} \ldots \mu_{p}}\right),
$$

where the field strength of $A_{\mu_{1} \ldots \mu_{p}}$ is defined in the standard manner by $F_{\mu_{1} \ldots \mu_{p+1}} \equiv \partial_{\left[\mu_{1}\right.} A_{\left.\mu_{2} \ldots \mu_{p+1}\right]}$. We use the Minkowski metric tensor of 'mostly minus' signature $\sigma_{\mu v}=\sigma^{\mu v}=\operatorname{diag}(+-\ldots-)$. The canonical analysis of the model described by the Lagrangian action (5) displays the constraints

$$
\chi^{(1) i_{1} \ldots i_{p-1}} \equiv \pi^{0 i_{1} \ldots i_{p-1}} \approx 0, \quad \chi_{i_{1} \ldots i_{p-1}}^{(2)} \equiv p \partial^{j} \pi_{j i_{1} \ldots i_{p-1}}-\frac{m^{2}}{(p-1) !} A_{0 i_{1} \ldots i_{p-1}} \approx 0
$$

and the canonical Hamiltonian

$$
\begin{aligned}
H_{c} & =\int d^{D-1} x\left(-\frac{p !}{2} \pi_{i_{1} \ldots i_{p}} \pi^{i_{1} \ldots i_{p}}-p A_{0 i_{1} \ldots i_{p-1}} \partial_{j} \pi^{j i_{1} \ldots i_{p-1}}\right. \\
& \left.+\frac{1}{2 \cdot(p+1) !} F_{i_{1} \ldots i_{p+1}} F^{i_{1} \ldots i_{p+1}}+\frac{m^{2}}{2 \cdot p !} A_{\mu_{1} \ldots \mu_{p}} A^{\mu_{1} \ldots \mu_{p}}\right)
\end{aligned}
$$


where $\pi^{\mu_{1} \ldots \mu_{p}}$ are the canonical momenta conjugated to the fields $A_{\mu_{1} \ldots \mu_{p}}$. Constraints (6) are second-class and irreducible [6].

In the sequel we apply the BF method exposed in the above to the case of massive $p$ forms. In view of this, we enlarge the original phase-space by adding the bosonic fields/momenta $\left\{B^{\mu_{1} \ldots \mu_{p-1}}, \Pi_{\mu_{1} \ldots \mu_{p-1}}\right\}$. The constraints $G_{A}(z, \zeta) \approx 0$ gain in the case of massive $p$-forms the concrete form

$$
\begin{gathered}
G^{(1) i_{1} \ldots i_{p-1}} \equiv \chi^{(1) i_{1} \cdots i_{p-1}}+m B^{i_{1} \ldots i_{p-1}} \approx 0, \quad G_{i_{1} \ldots i_{p-2}} \equiv \Pi_{0 i_{1} \ldots i_{p-2}} \approx 0, \\
G_{i_{1} \ldots i_{p-1}}^{(2)} \equiv \chi_{i_{1} \ldots i_{p-1}}^{(2)}-\frac{m}{(p-1) !} \Pi_{i_{1} \ldots i_{p-1}} \approx 0 .
\end{gathered}
$$

Constraints (8)-(9) form an Abelian and irreducible first-class constraint set. The first-class Hamiltonian complying with the general requirements (3) is expressed by

$$
\begin{gathered}
H_{\mathrm{BF}}=H_{c}+\int d^{D-1} x\left[\frac{1}{2(p-1) !} \Pi^{i_{1} \ldots i_{p-1}} \Pi_{i_{1} \ldots i_{p-1}}-\frac{1}{m} \Pi^{i_{1} \ldots i_{p-1}}\left(p \partial^{j} \pi_{j i_{1} \ldots i_{p-1}}-\frac{m^{2}}{(p-1) !} A_{0 i_{1} \ldots i_{p-1}}\right)\right. \\
\left.-\frac{1}{p}\left(m A^{i_{1} \ldots i_{p}}-\frac{(p-1) !}{2} \partial^{\left[i_{1}\right.} B^{\left.i_{2} \ldots i_{p}\right]}\right) \partial_{\left[i_{1}\right.} B_{\left.i_{2} \ldots i_{p}\right]}-\frac{p}{(p-1) !} B^{0 i_{1} \ldots i_{p-2}} \partial^{j}\left(m A_{0 j i_{1} \ldots i_{p-2}}+\Pi_{j i_{1} \ldots i_{p-2}}\right)\right] .
\end{gathered}
$$

\section{Covariant path integral for the first-class system. Topological coupling}

In the sequel we show how massive $p$-forms get related to $(D-p-1)$-form gauge fields. In order to do this, we start from the first-class system constructed in the above (subject to the first-class constraints (8)-(9), whose evolution is governed by the first-class Hamiltonian (10)). Imposing the canonical gauge conditions

$$
C_{j_{1} \cdots j_{p-1}}^{(1)} \equiv A_{0 j_{1} \cdots j_{p-1}} \approx 0, \quad C^{j_{1} \cdots j_{p-2}} \equiv B^{0 j_{1} \cdots j_{p-2}} \approx 0,
$$

we obtain that (8) and (11) generate a second-class constraint subset, while (9) is first-class. Eliminating the second-class constraints (8) and (11) (the coordinates of the reduced phasespace are $\left\{A_{i_{1} \ldots i_{p}}, B^{i_{1} \ldots i_{p-1}}, \pi^{i_{1} \ldots i_{p}}, \Pi_{i_{1} \ldots i_{p-1}}\right\}$, we are left with a system subject only to the first-class constraints

$$
G_{i_{1} \ldots i_{p-1}} \equiv p \partial^{j} \pi_{j i_{1} \ldots i_{p-1}}-\frac{m}{(p-1) !} \Pi_{i_{1} \ldots i_{p-1}} \approx 0,
$$

while the first-class Hamiltonian (10) takes the form

$$
\begin{gathered}
H_{\mathrm{BF}}=\int d^{D-1} x\left[-\frac{p !}{2} \pi_{i_{1} \ldots i_{p}} \pi^{i_{1} \ldots i_{p}}+\frac{1}{2 \cdot(p+1) !} F_{i_{1} \ldots i_{p+1}} F^{i_{1} \ldots i_{p+1}}+\frac{m^{2}}{2 \cdot p !} A_{i_{1} \ldots i_{p}} A^{i_{1} \ldots i_{p}}\right. \\
\left.+\frac{1}{2(p-1) !} \Pi^{i_{1} \ldots i_{p-1}} \Pi_{i_{1} \ldots i_{p-1}}-\frac{1}{m} \Pi^{i_{1} \ldots i_{p-1}} p \partial^{j} \pi_{j i_{1} \ldots i_{p-1}}-\frac{1}{p}\left(m A^{i_{1} \ldots i_{p}}-\frac{(p-1) !}{2} \partial^{\left[i_{1}\right.} B^{\left.i_{2} \ldots i_{p}\right]}\right) \partial_{\left[i_{1}\right.} B_{\left.i_{2} \ldots i_{p}\right]}\right] .
\end{gathered}
$$

We consider the quantities 


$$
\Phi_{i_{1} \ldots i_{p}}=A_{i_{1} \ldots i_{p}}-\frac{(p-1) !}{m} \partial_{\left[i_{1}\right.} B_{\left.i_{2} \ldots i_{p}\right]}, \quad \Phi_{0 i_{1} \ldots i_{p-1}}=\frac{1}{m} \Pi_{i_{1} \ldots i_{p-1}},
$$

which are in (strong) involution with first-class constraints (12). We define

$$
\Omega_{\mu_{1} \ldots \mu_{p+1}}=\partial_{\left[\mu_{1}\right.} \Phi_{\left.\mu_{2} \ldots \mu_{p+1}\right]} \text {, }
$$

where $\Phi_{\mu_{1} \ldots \mu_{p}} \equiv\left\{\Phi_{0 i_{1} \ldots i_{p-1}}, \Phi_{i_{1} \ldots i_{p}}\right\}$. By direct computation, it follows that

$$
\partial^{v} \Omega_{v \mu_{1} \ldots \mu_{p}}=m^{2} \Phi_{\mu_{1} \ldots \mu_{p}}+\mathrm{O}\left(G_{i_{1} \ldots i_{p-1}}\right) .
$$

From (16) we obtain that the equalities

$$
\partial^{v} \Phi_{v \mu_{1} \ldots \mu_{p-1}}=0
$$

hold on the first-class surface (12). The solution to (16) is of the type

$$
\Phi_{\mu_{1} \ldots \mu_{p}}=-\frac{1}{m} \frac{1}{(D-p) !} \varepsilon_{\mu_{1} \ldots \mu_{p} v_{1} \ldots v_{D-p}} \partial^{\left[v_{1}\right.} V^{\left.v_{2} \ldots \nu_{D-p}\right]} .
$$

Consequently, we enlarge the phase-space by adding the bosonic fields/momenta $\left\{V^{\nu_{1} \cdots \nu_{D-p-1}}\right.$, $\left.P_{v_{1} \ldots v_{D-p-1}}\right\}$. If we replace (18) in (12), then the constraint set takes the form

$$
p \partial^{j} \pi_{j i_{1} \ldots i_{p-1}}+\frac{m}{(p-1) !(D-p) !} \varepsilon_{0 i_{1} \ldots i_{p-1} j_{1} \ldots j_{D-p}} \partial^{\left[j_{1}\right.} V^{\left.j_{2} \ldots j_{D-p}\right]} \approx 0,
$$

remains first-class, and becomes reducible of order $(p-1)$. In order to preserve the number of physical degrees of freedom we must impose the supplementary constraints

$$
P_{0 i_{1} \ldots i_{D-p-2}} \approx 0, \quad(-)^{D-p-1}(D-p-1) \partial^{j} P_{j i_{1} \ldots i_{D-p-2}} \approx 0 .
$$

The constraints (19) and (20) are first-class and reducible of order $D-p-2$ (or $p-1$ if $D-p-1 \leq p)$. The gauge transformation of the quantity $\partial_{\left[i_{1}\right.} B_{\left.i_{2} \ldots i_{p}\right]}$ leads to the relation

$$
\partial_{\left[i_{1}\right.} B_{\left.i_{2} \ldots i_{p}\right]}=\frac{(-)^{D-p-1}}{(p-1) !} \varepsilon_{0 i_{1} \ldots i_{p} j_{1} \ldots j_{D-p-1}} P^{j_{1} \cdots j_{D-p-1}} .
$$

For each first-class theory we can identify a set of fundamental classical observables such that they are in a one-to-one correspondence and possess the same Poisson brackets. The procedure exposed previously preserves the equivalence between the two first-class theories. As a result, the BF theory and the reducible first-class system remain equivalent also at the level of the Hamiltonian path integral quantization. This further implies that the reducible first-class system is completely equivalent with the original second-class theory. Due to this equivalence one can replace the Hamiltonian path integral of massive $p$-forms with that associated with the first-class system reducible of order $D-p-2$ (or $p-1$ if $D-p-1 \leq p$ ). Using (14), (18), (21) and the first-class Hamiltonian (13) we obtain that the argument of the exponential from the Hamiltonian path integral of the reducible first-class system as 


$$
\begin{gathered}
S_{\mathrm{BF}}=\int d^{D} x\left[\left(\partial_{0} A_{i_{1} \ldots i_{p}}\right) \pi^{i_{1} \ldots i_{p}}+\left(\partial_{0} V_{0 i_{1} \ldots i_{D-p-2}}\right) P^{0 i_{1} \ldots i_{D-p-2}}+\left(\partial_{0} V_{i_{1} \ldots i_{D-p-1}}\right) P^{i_{1} \ldots i_{D-p-1}}\right. \\
+\frac{p !}{2} \pi_{i_{1} \ldots i_{p}} \pi^{i_{1} \ldots i_{p}}-\frac{1}{2 \cdot(p+1) !} F_{i_{1} \ldots i_{p+1}} F^{i_{1} \ldots i_{p+1}}-(-)^{D} \frac{1}{2 \cdot(D-p) !} \partial_{\left[_{1}\right.} V_{\left.i_{2} \ldots i_{D-p}\right]} \partial^{\left[i_{1}\right.} V^{\left.i_{2} \ldots i_{D-p}\right]} \\
-\frac{m^{2}}{2 \cdot p !} A_{i_{1} \ldots i_{p}} A^{i_{1} \ldots i_{p}}-(-)^{D-p} \frac{m}{p !} \varepsilon_{0 i_{1} \ldots i_{p} j_{1} \ldots j_{D-p-1}} A^{i_{1} \ldots i_{p}} P^{j_{1} \ldots j_{D-p-1}}+(-)^{D} \frac{(D-p-1) !}{2} P_{i_{1} \ldots i_{D-p-1}} P^{i_{1} \ldots i_{D-p-1}} \\
-(-)^{p} \bar{\lambda}_{i_{1} \ldots i_{p-1}}\left(p \partial_{j} \pi^{j i_{1} \ldots i_{p-1}}+\frac{m}{(p-1) \cdot(D-p) !} \varepsilon^{0 i_{1} \ldots i_{p-1} k_{1} \ldots k_{D-p}} \partial_{k_{1}} V_{\left.k_{2} \ldots k_{D-p}\right]}\right) \\
\left.-\lambda_{i_{1} \ldots i_{D-p-2}}^{(1)} P^{0 i_{1} \ldots i_{D-p-2}}-(-)^{D-p-1}(D-p-1) \lambda_{i_{1} \ldots i_{D-p-2}}^{(2)} \partial_{j} P^{j i_{1} \ldots i_{D-p-2}}\right]
\end{gathered}
$$

where $\bar{\lambda}_{i_{1} \ldots i_{p-1}}=(-)^{p}\left(\lambda_{i_{1} \ldots i_{p-1}}-\frac{1}{m} \frac{1}{(D-p) !} \varepsilon_{0 i_{1} \ldots i_{p-1} j_{1} \ldots j_{D-p}} \partial^{j_{1}} V^{\left.j_{2} \ldots j_{D-p}\right]}\right)[7]-[8]$.

In order to obtain from (22) a path integral exhibiting a manifestly Lorentz-covariant functional in its exponential, we enlarge the original phase-space with the Lagrange multipliers $\left\{\bar{\lambda}_{i_{1} \ldots i_{p-1}}, \lambda_{i_{1} \ldots i_{D-p-2}}^{(2)}\right\}$ and their canonical momenta $\left\{\pi^{i_{1} \ldots i_{p-1}}, p^{i_{1} \ldots i_{D-p-2}}\right\}$ and add the constraints

$$
\pi^{i_{1} \cdots i_{p-1}} \approx 0 \quad p^{i_{1} \ldots i_{D-p-2}} \approx 0 .
$$

Performing in the path integral some partial integrations over the auxiliary fields and using the notations

$$
\bar{\lambda}_{i_{1} \ldots i_{p-1}} \equiv \bar{A}_{i_{1} \ldots i_{p-1} 0}, \quad \lambda_{i_{1} \ldots i_{D-p-2}}^{(2)} \equiv \bar{V}_{i_{1} \ldots i_{D-p-2} 0},
$$

the argument of the exponential becomes

$$
\begin{gathered}
S_{\mathrm{BF}}=\int d^{D} x\left[-\frac{1}{2 \cdot(p+1) !} F_{i_{1} \ldots i_{p+1}} F^{i_{1} \ldots i_{p+1}}-\frac{1}{2 \cdot p !} \bar{F}_{0 i_{1} \ldots i_{p}} \bar{F}^{0 i_{1} \ldots i_{p}}-(-)^{D} \frac{1}{2 \cdot(D-p) !} F_{i_{1} \ldots i_{D-p}} F^{i_{1} \ldots i_{D-p}}\right. \\
-(-)^{D} \frac{1}{2 \cdot(D-p-1) !} F_{0 i_{1} \ldots i_{D-p-1}} F^{0 i_{1} \ldots i_{D-p-1}}+\frac{m}{(p-1) !(D-p) !} \varepsilon_{0 i_{1} \ldots i_{p-1} j_{1} \ldots j_{D-p}} \bar{A}^{0 i_{1} \ldots i_{p-1}} F^{j_{1} \ldots j_{D-p}} \\
\left.+\frac{m}{p !(D-p-1) !} \varepsilon_{i_{1} \ldots i_{p} 0 j_{1} \ldots j_{D-p-1}} A^{i_{1} \ldots i_{p}} F^{0 j_{1} \ldots j_{D-p-1}}\right] .
\end{gathered}
$$

In the last functional we also used the notations

$$
\begin{gathered}
\bar{F}_{0 i_{1} \ldots i_{p}}=\partial_{0} A_{i_{1} \ldots i_{p}}+(p-1)^{p} \partial_{\left[i_{1}\right.} \bar{A}_{\left.i_{2} \ldots i_{p}\right] 0}, \quad F_{i_{1} \ldots i_{D-p}}=\partial_{\left[i_{1}\right.} V_{\left.i_{2} \ldots i_{D-p}\right]}, \\
F_{0 i_{1} \ldots i_{D-p-1}}=\partial_{0} V_{i_{1} \ldots i_{D-p-1}}+(-)^{D-p-1} \partial_{\left[i_{1}\right.} \bar{V}_{\left.i_{2} \ldots i_{D-p-1}\right] 0} .
\end{gathered}
$$

The functional (25) associated with the reducible first-class system takes a manifestly Lorentz-covariant form

$$
\begin{gathered}
S_{\mathrm{BF}}=\int d^{D} x\left[-\frac{1}{2 \cdot(p+1) !} \bar{F}_{\mu_{1} \ldots \mu_{p+1}} \bar{F}^{\mu_{1} \ldots \mu_{p+1}}-(-)^{D} \frac{1}{2 \cdot(D-p) !} F_{\mu_{1} \ldots \mu_{D-p}} F^{\mu_{1} \ldots \mu_{D-p}}\right. \\
\left.+\frac{m}{p !(D-p) !} \varepsilon_{\mu_{1} \ldots \mu_{p} \nu_{1} \ldots \nu_{D-p}} \bar{A}^{\mu_{1} \ldots \mu_{p}} F^{\nu_{1} \ldots \nu_{D-p}}\right]
\end{gathered}
$$


and describes a topological coupling between the $p$-form $\bar{A}_{\mu_{1} \ldots \mu_{p}}$ and the $(D-p-1)$-form $\bar{V}_{\mu_{1} \ldots \mu_{p+1}}[9]-[10]$.

\section{Conclusions}

In this paper we performed the path integral quantization of massive p-forms in the framework of BF method. The BF approach involves an appropriate extension of the original phase-space and then the construction of a first-class system on the extended phase-space that reduces to the original, second-class theory if one sets all the extravariables equal to zero. Based on an appropriate extension of the phase-space, integrating out the auxiliary fields and performing some field redefinitions, we find the manifestly Lorentz-covariant path integrals corresponding to the Lagrangian formulation of the first-class systems which reduce to the Lagrangian path-integral for $p$ - and $(D-p-1)$-forms with topological coupling.

\section{Acknowledgements}

One of the authors (S.C.S.) was supported by the strategic grant POSDRU/89/1.5/S/61968, Project ID61968 (2009), co-financed by the European Social Fund within the Sectorial Operational Program Human Resources Development 2007-2013.

\section{References}

[1] I. A. Batalin and E. S. Fradkin,Phys. Lett. B180 (1986) 157

[2] I. A. Batalin and E. S. Fradkin, Phys. Lett. B236 (1990) 528

[3] I. A. Batalin and E. S. Fradkin, Nucl. Phys. B279 (1987) 514

[4] I. A. Batalin and I. V. Tyutin, Int. J. Mod. Phys. A6 (1991) 3255

[5] C. Bizdadea, J. Phys. A: Math. Gen. 29 (1996) 3985

[6] M. Henneaux and C. Teitelboim, Quantization of Gauge Systems, Princeton University Press, (1992)

[7] E. M. Cioroianu, S. C. Sararu and O. Balus, Int. J. Mod. Phys. A25 (2010) 185

[8] S. C. Sararu, Massive p-forms:first-class approaches, (2011) in preparation

[9] E. Cremmer and J. Scherk, Nucl. Phys. B72 (1974) 117

[10] C. Bizdadea, E. M. Cioroianu and S. O. Saliu, Phys. Scripta 60 (1999) 120 